\title{
CXCL10 Is a Circulating Inflammatory Marker in Patients with Advanced Heart Failure: a Pilot Study
}

Citation for published version (APA):

Altara, R., Manca, M., Hessel, M. H., Gu, Y., van Vark, L. C., Akkerhuis, K. M., Staessen, J. A., Struyker Boudier, H., Booz, G. W., \& Blankesteijn, M. (2016). CXCL10 Is a Circulating Inflammatory Marker in Patients with Advanced Heart Failure: a Pilot Study. Journal of Cardiovascular Translational Research, 9(4), 302-314. https://doi.org/10.1007/s12265-016-9703-3

Document status and date:

Published: 01/08/2016

DOI:

10.1007/s12265-016-9703-3

Document Version:

Publisher's PDF, also known as Version of record

Document license:

Taverne

Please check the document version of this publication:

- A submitted manuscript is the version of the article upon submission and before peer-review. There can be important differences between the submitted version and the official published version of record.

People interested in the research are advised to contact the author for the final version of the publication, or visit the DOI to the publisher's website.

- The final author version and the galley proof are versions of the publication after peer review.

- The final published version features the final layout of the paper including the volume, issue and page numbers.

Link to publication

\footnotetext{
General rights rights.

- You may freely distribute the URL identifying the publication in the public portal. please follow below link for the End User Agreement:

www.umlib.nl/taverne-license

Take down policy

If you believe that this document breaches copyright please contact us at:

repository@maastrichtuniversity.nl

providing details and we will investigate your claim.
}

Copyright and moral rights for the publications made accessible in the public portal are retained by the authors and/or other copyright owners and it is a condition of accessing publications that users recognise and abide by the legal requirements associated with these

- Users may download and print one copy of any publication from the public portal for the purpose of private study or research.

- You may not further distribute the material or use it for any profit-making activity or commercial gain

If the publication is distributed under the terms of Article $25 \mathrm{fa}$ of the Dutch Copyright Act, indicated by the "Taverne" license above, 


\title{
CXCL10 Is a Circulating Inflammatory Marker in Patients with Advanced Heart Failure: a Pilot Study
}

\author{
Raffaele Altara ${ }^{1,2}$ - Marco Manca $^{3}$ - Marleen H. Hessel ${ }^{4}$ - Yumei Gu ${ }^{5,6}$. \\ Laura C. van Vark $^{7}$ - K. Martijn Akkerhuis ${ }^{7}$. Jan A. Staessen ${ }^{5,6}$. \\ Harry A. J. Struijker-Boudier ${ }^{1}$ - George W. Booz ${ }^{2}$-W. Matthijs Blankesteijn ${ }^{1}$
}

Received: 25 March 2016 / Accepted: 30 May 2016/Published online: 6 June 2016

(C) Springer Science+Business Media New York 2016

\begin{abstract}
Chemokines are involved in the remodeling of the heart; however, their significance as biomarkers in heart failure is unknown. We observed that circulating CXCR3 receptor chemokines CXCL9 and CXCL10 in a rat model of heart failure were increased 1 week after myocardial infarction. CXCL10 was also increased in both remote and infarcted regions of the heart and remained elevated at 16 weeks; CXCL9 was elevated in the remote area at 1 week. In humans, hierarchical clustering and principal component analysis revealed that circulating CXCL10, MIP-1 $\alpha$, and CD40 ligand were the best indicators for differentiating healthy and heart failure subjects. Serum CXCL10 levels were increased in
\end{abstract}

Associate Editor Enrique Lara-Pezzi oversaw the review of this article

Electronic supplementary material The online version of this article (doi:10.1007/s12265-016-9703-3) contains supplementary material,

which is available to authorized users.

Raffaele Altara

raltara@umc.edu

1 Department of Pharmacology, Cardiovascular Research Institute Maastricht (CARIM), Maastricht University, Maastricht, The Netherlands

2 Department of Pharmacology and Toxicology, School of Medicine, University of Mississippi Medical Center, 2500 North State St., Jackson, MS 39216-4505, USA

3 DG-DI, Medical Applications, CERN, Geneva, Switzerland

4 Leiden University Medical Center, Leiden, The Netherlands

5 Studies Coordinating Centre, Research Unit Hypertension, Leuven, Belgium

6 Cardiovascular Epidemiology, KU Leuven Department of Cardiovascular Sciences, University of Leuven, Leuven, Belgium

7 Department of Cardiology, Thoraxcenter, Erasmus MC, Rotterdam, The Netherlands patients with symptomatic heart failure as indexed by NYHA classification II through IV. The presence of CXCL10, MIP-1 $\alpha$, and CD40 ligand appears to be dominant in patients with advanced heart failure. These findings identify a distinct profile of inflammatory mediators in heart failure patients.

Keywords Chemokines · Inflammation · Immune response · CXCR3

\begin{tabular}{|c|c|}
\hline \multicolumn{2}{|l|}{ Abbreviations } \\
\hline ACCF/AHA & American College of Cardiology \\
\hline & Foundation/American Heart Association \\
\hline BNP & B-type natriuretic peptide \\
\hline $\mathrm{CD} 40$ & Cluster of differentiation 40 \\
\hline CXCL & Chemokine (C-X-C motif) ligand \\
\hline CXCR & Chemokine (C-X-C motif) receptor \\
\hline FLEMENGHO & $\begin{array}{l}\text { Flemish Study on Environment, Genes, } \\
\text { and Health Outcomes }\end{array}$ \\
\hline $\mathrm{HF}$ & Heart failure \\
\hline IFN- $\gamma$ & Interferon gamma \\
\hline IL & Interleukin \\
\hline IP-10 & Interferon gamma-induced protein 10 \\
\hline LAD & Left anterior descending artery \\
\hline $\mathrm{LV}$ & Left ventricle/ventricular \\
\hline MCP1 & Monocyte chemoattractant protein 1 \\
\hline MI & Myocardial infarction \\
\hline MIF & Macrophage migration inhibitory factor \\
\hline MIP- $1 \alpha$ & Macrophage inflammatory protein- 1 alpha \\
\hline NYHA & New York Heart Association \\
\hline NT-proBNP & $\begin{array}{l}\text { N-terminal of the prohormone brain natri- } \\
\text { uretic peptide }\end{array}$ \\
\hline PCA & Principal component analysis \\
\hline SDF1 & Stromal cell-derived factor 1 \\
\hline TIMP-1 & Tissue inhibitor of metalloproteinases-1 \\
\hline $\mathrm{TNF} \alpha$ & Tumor necrosis factor alpha \\
\hline
\end{tabular}


TRIUMPH TRanslational Initiative on Unique and novel strategies for Management of Patients with Heart failure

\section{Introduction}

The global heart failure epidemic has been escalating for decades and is a leading cause of both hospitalization and death in the western world [1]. A significant bottleneck in cardiovascular care is the insufficient availability of robust tools for prognosis, diagnosis, and therapeutics of heart failure. To address this need, the identification of biomarkers may spur the development of novel strategies for management of patients.

In 1928, the New York Heart Association (NYHA) proposed a scoring system to classify the severity of heart failure in patients based on clinical symptoms and prognosis. Since then, appropriate revisions have been made based on best medical practice to provide the most accurate assessment of patient status. In the last two decades, a series of biomarkers have been introduced in clinical practice in order to improve the classification and management of heart failure patients [2-5]. To a considerable extent, the natriuretic peptides BNP and NT-proBNP provide critical utility so far as confirming the diagnosis of heart failure in suspected patients or classifying the degree of heart failure severity according to the ACCF/ AHA stages of heart failure and the NYHA functional classifications [6]. However, biological variability in blood peptide levels may confound their clinical application, and their use in monitoring or adjusting drug therapy is uncertain [7-11]. In addition, not all heart failure cases can be easily characterized according to ACCF/AHA or NYHA guidelines [7], and some patients may have falsely low BNP or NT-proBNP levels due to obesity or heart failure with preserved ejection fraction [7, $12,13]$. Therefore, there is a need for additional biomarkers to stratify heart failure patients.

Recently, we reported the finding of elevated levels of the CXCR3 agonists, CXCL-9, -10, and -11 at an early stage of heart failure in mice that had undergone transverse aortic constriction (TAC) and in patients with hypertension associated with subclinical or symptomatic diastolic left ventricular dysfunction $[14,15]$. In the present study, we present experimental evidence for the sustained activation of an inflammatory profile for CXCL10 in an animal model of heart failure from myocardial infarction (MI). The chemokine CXCL10, also known as interferon gamma-induced protein 10 (IP-10), is critically involved in the inflammatory response as a chemoattractant for Th1 cells, monocytes/macrophages, NK cells, and dendritic cells, and as a promotor of $\mathrm{T}$ cell adhesion to endothelial cells [15]. Moreover, we determined that circulating levels of CXCL10 are elevated in patients with most advanced end-stage heart failure of both ischemic and nonischemic etiology.

\section{Methods}

\section{Myocardial Infarction (MI) in Rat}

All experimental and surgical procedures were approved by the Institutional Council on Animal Care and Use of the Maastricht University and complied with the National Institutes of Health Guide for the Care and Use of Laboratory Animals. MI was induced in 3-month-old male Wistar rats (Harlan), under isoflurane anesthesia by permanent ligation of the left descending coronary artery, as previously described [16]. Only rats with an infarct size $>30 \%$ of the total endocardial circumference were included in the study. Infarct size averaged $46 \pm 6 \%$ of the total left ventricle $(n=8)$. As reported [17], ejection fraction (EF) declines after 16 weeks in the rat MI model to $<40 \%$, symptomatic of heart failure. Animals were sacrificed at 1 and 16 weeks after operation. Control rats underwent sham surgery.

\section{Rat Sample Preparation}

Serum Blood was sampled from the abdominal aorta during sacrifice and was allowed to clot for $4 \mathrm{~h}$ at $4{ }^{\circ} \mathrm{C}$ before centrifuging for $10 \mathrm{~min}$ at $2000 \mathrm{~g}$. Serum was removed and immediately aliquoted and stored (for 6 months on average) at $<-80{ }^{\circ} \mathrm{C}$.

Tissue Lysate Immediately after the animals were sacrificed by bleeding, hearts were excised, weighed, and divided into left ventricle (LV), right ventricle (RV), and atria for further analysis. The LV were homogenized in protease inhibitor cocktail (Complete Mini Protease Inhibitor Cocktail tablets, Roche) and kept on ice as much as possible to avoid deterioration. The lysed tissues were then centrifuged at $10,000 \mathrm{~g}$ for $5 \mathrm{~min}$ at $4{ }^{\circ} \mathrm{C}$ to remove cellular debris. The supernatant was collected for analysis.

\section{Human Study Population}

For the purpose of this study, three cohorts were used. One cohort was composed of healthy individuals. Twenty-nine healthy volunteers (15 males and 14 females) above 50 years of age were recruited under the supervision of a physician or cardiologist who overall judged whether each individual fulfilled the general criteria for a healthy person. The subjects were pre-screened and excluded in the case of an ongoing chronic inflammatory disease, recent illness of any kind in the last 3 months, or use of anti-inflammatory drugs. Volunteers were scheduled for blood withdrawal in the morning between 9 and $10 \mathrm{am}$. The second cohort consisted of 28 heart failure patients hospitalized with decompensation of known chronic HF or newly diagnosed HF. The personal medical history and the blood samples 
of the heart failure patients were collected by the TRIAL ID: NTR1893, TRanslational Initiative on Unique and novel strategies for Management of Patients with Heart failure (TRIUMPH), The Netherlands. Table 1 lists the most prominent features used to analyze the groups. The recruitment of subjects was performed according to the Medical Ethical Committees of Groningen and Maastricht University, The Netherlands, and in accordance with of the Declaration of Helsinki. The third cohort was extracted from FLEMENGHO, a large-scale family-based and nested-case-control study on the genetic epidemiology of cardiovascular phenotypes. Based on age and sex match, we selected 26 cases: 17 asymptomatic (NYHA class I) and 9 symptomatic (3 NYHA class II and 6 NYHA class III) for left ventricular dysfunction and 31 normotensive healthy controls who underwent echocardiography be- tween 2005 and 2010. The recommendations of the American Society of Echocardiography for data acquisition and offline analysis were followed [18, 19]. Patients were assigned to NYHA class based on clinical data, including NT-proBNP levels.

\section{Patient Sample Collection}

Non-fasting blood samples were obtained by venipuncture and transported to the clinical chemistry laboratory of each participating hospital for further processing according to a standardized protocol. The collected material was centrifuged at $1700 \mathrm{~g}$. Blood serum was collected. All blood aliquots were subsequently stored at a temperature of $-80^{\circ} \mathrm{C}$ within $2 \mathrm{~h}$ after venipuncture.

Table 1 Biometrical data of TRIUMPH participants

\begin{tabular}{|c|c|c|c|c|}
\hline & Healthy & NYHA II & NYHA III & NYHA IV \\
\hline Male & 15 & 4 & 5 & 4 \\
\hline Female & 14 & 4 & 10 & 1 \\
\hline Age & $59 \pm 1.1$ & $53 \pm 4.3$ & $54 \pm 1.7$ & $45 \pm 6.5$ \\
\hline Etiology & NA & 1 ischemic, 7 non-ischemic & 2 ischemic, 13 non-ischemic & 3 ischemic, 2 non-ischemic \\
\hline Primary cause HF (not ischemic) & NA & $\mathrm{CM}$, hypertension, $\mathrm{CHD}$ & Hypertension, CM, CHD, VD, arrhythmias & $\mathrm{CM}$ \\
\hline Type of cardiomyopathy & NA & DCM, other & DCM, HCM & DCM, HCM \\
\hline Hypertension & NA & 2 & 6 & 1 \\
\hline Myocardial infarction & NA & 0 & 4 & 2 \\
\hline Congenital heart defect & NA & 1 & 0 & 0 \\
\hline COPD & NA & 1 & 3 & 2 \\
\hline Chronic renal failure & NA & 1 & 8 & 0 \\
\hline Diabetes mellitus & NA & 1 & 7 & 0 \\
\hline Diabetes type & NA & NIDDM & NIDDM & \\
\hline Hypercholesterolemia & NA & 0 & 2 & 0 \\
\hline Dyspnea & NA & 3 & 19 & 4 \\
\hline Angina pectoris & NA & 1 & 3 & 0 \\
\hline $\mathrm{SBP}(\mathrm{mmHg})$ & ND & $132 \pm 9.8$ & $126.5 \pm 5.9$ & $102.8 \pm 7.8$ \\
\hline DBP (mmHg) & ND & $84 \pm 5.7$ & $80 \pm 4.5$ & $65.2 \pm 4.4$ \\
\hline $\mathrm{EF}(\%)$ & ND & $26.5 \pm 4.3$ & $26.3 \pm 3.4$ & $26.7 \pm 1.7$ \\
\hline Hemoglobin $(\mathrm{mmol} / \mathrm{L})$ & ND & $8.7 \pm 0.8$ & $7.9 \pm 0.3$ & $11.5 \pm 0.9$ \\
\hline Creatinine $(\mathrm{mmol} / \mathrm{L})$ & ND & $100.3 \pm 15.6$ & $148.9 \pm 21.7$ & $72.5 \pm 9.8$ \\
\hline Uricemia $(\mathrm{mmol} / \mathrm{L})$ & ND & $9.7 \pm 1.1$ & $21.95 \pm 5.4$ & $5.3 \pm 0.1$ \\
\hline CRP (mg/L) & ND & $12.2 \pm 3.7$ & $10.7 \pm 2.5$ & $67.7 \pm 61.7$ \\
\hline NT-proBNP (pg/mL) & ND & $1784 \pm 1407$ & $2007 \pm 847.1$ & $11,034 \pm 6613$ \\
\hline CXCL10 (pg/mL) & $98.9 \pm 10.1$ & $237.5 \pm 50.8^{* *}$ & $207 \pm 36.7 * *$ & $145.5 \pm 56.2$ \\
\hline
\end{tabular}

The descriptive information of the TRIUMPH cohort is reported here. Patients were selected based on age and sex matching with the healthy individuals. NYHA classification ranges from II to IV. Values are indicated as the means of the group \pm the SEM. Statistical significance has been calculated by oneway ANOVA followed by Tukey's test (CI of $95 \%$ )

$N A$ not applicable, $C H D$ coronary heart disease, $C M$ cardiomyopathy, $C R P$ C-reactive protein, $N D$ not determined, $D C M$ dilated cardiomyopathy, $H C M$ hypertrophic cardiomyopathy, $N I D D M$ noninsulin-dependent diabetes mellitus, $C O P D$ chronic obstructive pulmonary disease, $S B P$ systolic blood pressure, $D B P$ diastolic blood pressure, $E F$ ejection fraction, $V D$ valve deviations 


\section{Multiple Cytokine Detection by Multiplex Membrane-Based Immunoassay}

Rat cytokine array panel A (R\&D Systems) was performed following the guidelines we recently described [20]. For serum evaluation, a total volume of $800 \mu \mathrm{l}$ was pooled using $200 \mu \mathrm{l}$ from each animal ( $4 \times$ animals per membrane). For rat heart lysates, homogenized tissues were quantified (BCA protein assay-Pierce) and then pooled generating equal amounts of protein content $(0.5 \mu \mathrm{g} / \mathrm{mL})$ for measurement with each membrane. Samples were run in duplicate. Variability in mean florescent intensity (MFI) between replicates was less than 0.02. The human cytokine array panel A (R\&D Systems) was used according to the manufacturer's instructions to determine the levels of 36 cytokines in a semi-quantitative way. Each membrane was incubated with $1000 \mu \mathrm{L}$ (maximum possible volume) of serum. The method used for fluorescence detection is described by Altara et al. [20]. The multiplex membrane-based immunoassay exhibits sensitivities and specificities comparable to the electrochemiluminescence clinical assay used to quantify plasma NT-pro-BNP [20, 21].

\section{CXCL10 (CXCL10) Detection via Enzyme-Linked Immunosorbent Assay (ELISA)}

The human CXCL10 Quantikine ELISA kit was purchased from R\&D Systems. The assay was performed according to the manufacturer's instructions and $100 \mu \mathrm{L}$ of serum was added to each well.

\section{Statistical Analysis}

For cytokine/chemokine profile analysis in human blood, raw data were imported in R. Background was subtracted from raw data for each membrane. LIMMA package, a software environment for statistical computing in $\mathrm{R}$, was used to generate heat maps and perform hierarchical cluster analysis. Principal component analysis (PCA), an unsupervised pattern recognition method, was employed to examine the intrinsic variation in the data set. The readouts of the membranes were tested for linear relationship with the predicted concentrations by use of the MASS package, "Modern Applied Statistics with S" (4th edition. 2002). Evaluation of the quality of predictions was performed by Global Validation of Linear Models Assumptions (gvlma). A ROUT test $(Q=1 \%)$ was performed to identify the serum CXCL10 value of one healthy individual as an outlier, and this value $(380.1 \mathrm{pg} / \mathrm{mL})$ was excluded from all further analysis. This individual reported a family history of heart disease. GraphPad statistical program was used for graphics, and statistical analysis of data was performed by ANOVA and an appropriate post hoc test for multiple comparisons or a Student's $t$ test for two comparisons. Values are expressed as mean \pm SEM (n). A $p$ value $\leq 0.05$ was considered statistically significant.

\section{Results}

\section{Identification of Novel Inflammatory Biomarkers in Rat MI Model}

In our study, we selected a time point of 1 week after MI surgery to ensure that the inflammatory pathway observed was not due to the acute phase related to the surgery. Two of the most upregulated inflammatory mediators detected in serum were CXCL9 and CXCL10 (Fig. 1). Together with CXCL9 and CXCL10, cytokines with the highest values that were already known to be involved in cardiac remodeling (i.e., TIMP-1, IL-17, and IL-1 $\beta$ ) are shown in Fig. 1. We further investigated whether the inflammatory pattern detected in the circulation resembled the one present in the heart tissue (Fig. 2). We examined tissue from both the infarcted and remote areas of the heart (Fig. 3). At 1 week, all five cytokines were upregulated in the remote area (RA), but only CXCL10, TIMP-1 and IL-1 $\beta$ were also higher in the infarcted tissue (Fig. 2a). Unlike the other inflammatory mediators, CXCL10 was higher in the infarct area compared to the remote area and remained elevated at 16 weeks (Fig. 2b). At 16 weeks, cardiac function as assessed by ejection fraction was markedly reduced in the MI group.

\section{Circulating Inflammatory Profile in Heart Failure Patients and Healthy Subjects}

The blood inflammatory profile of the subjects involved in this study ( 29 healthy and 28 patients from the 2 nd cohort)

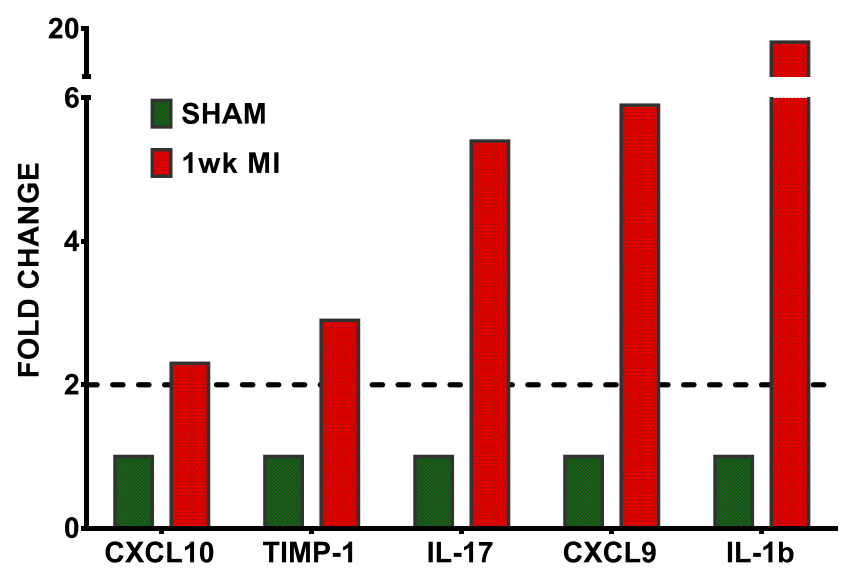

Fig. 1 Representative inflammatory profile of MI rats and shams. Serum was collected from rats 1 week after MI or sham operation and analyzed for inflammatory profile. A two-fold change is noted by a dashed line to facilitate evaluation of the data. CXCL10, TIMP-1, IL-17, MIG, and IL$1 \beta$ were upregulated in comparison to controls. Pooled samples of four rats were used in the assay. All analyses were done as replicates 


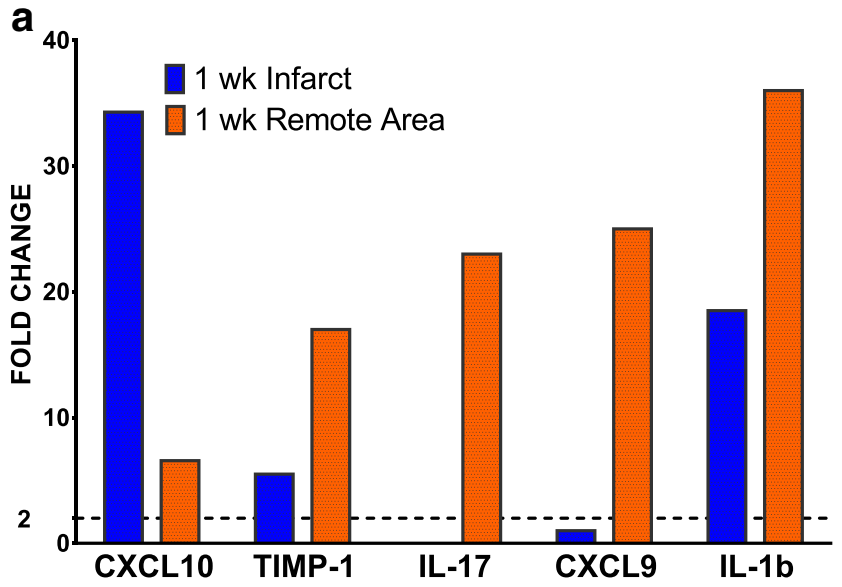

b

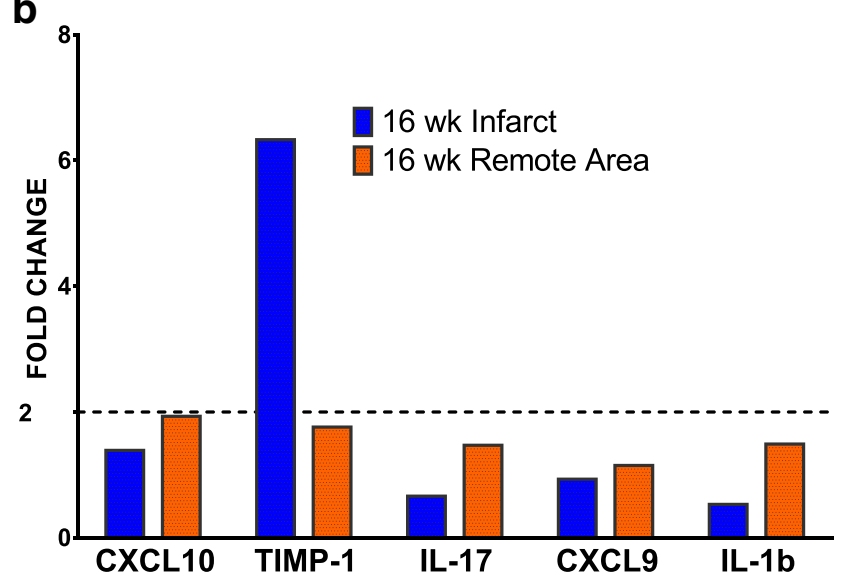

Fig. 2 Inflammatory mediators detected in tissue lysates of infarcted tissues, remote areas of MI rats, and septum of shams. Homogenized heart tissue (pooled from four rats) was assayed by membrane-based technique for inflammatory mediators. Remote areas $(R A)$, i.e., septum and infarcted regions were compared to healthy tissues from the shams. a Analysis was done 1 week post-MI. All five analytes shown were elevated in the RAs. CXCL10, TIMP-1, and IL-1 $\beta$ were also higher in the infarcted tissue compared to the sham RA. b Analysis was done at 16 weeks. CXCL10 remained moderately elevated in the RA. A two-fold change is noted by a dashed line to facilitate evaluation of the data. All analyses were done as replicates was determined using the adapted proteome profile protocol described in Altara et al. [20]. In order to extract information from the large data set that we obtained (57 subjects multiplied by the 36 determined cytokines, all in duplicate) and to transform it into an understandable structure, divisive hierarchical clustering was applied and a heat map generated (Fig. 4). The outcome is represented by two dendrograms (on top and on the left side of the figure) showing that all observations started in one cluster, and splits generated as one moves recursively down the hierarchy. As indicated by the "blue" (heart failure patients) and "peach" (controls, i.e., healthy subjects) colored bars, on the right side of Fig. 4, the two groups separated into three subgroups, with healthy subjects compactly positioned in the center of the chart. Considering the intensity of the color key used for the heat map, the inflammatory mediators Serpin E1, Sicam-1, RANTES, and MIF had the highest values for both groups. Regardless of those high intensities, the general intensity determined in the healthy subjects was much lower when compared to the patients, indicating much more pronounced inflammation in the patient group. Similar to the analysis performed in the rat MI model, the top seven cytokines in terms of fold-change are shown in Supplementary Figure 1.

\section{Multivariate Analysis}

Through orthogonal transformation of all values obtained by the membrane-based technology, we revealed the internal structure of the data using PCA in a way that best explains variance. We could confirm that heart failure patients and healthy subjects separated into two distinct groups based on inflammatory profile (Fig. 5). The first principal component, which accounts for as much of the variability in the data as possible, showed that MIP- $1 \alpha$ (macrophage inflammatory protein- $1 \alpha$ ) and CXCL10 were the best predictors for separation of the groups. The second component, which had the highest possible variance under the constraint that is
Fig. 3 Quantification of infarct size. Azan staining of cardiac sections was done at 1 week and 16 weeks after MI. Right panels: Heart tissue samples for cytokine profiling were taken from the infarcted $(I)$ and remote $(R)$ areas. Shown also is the left ventricular area at risk $(A A R)$

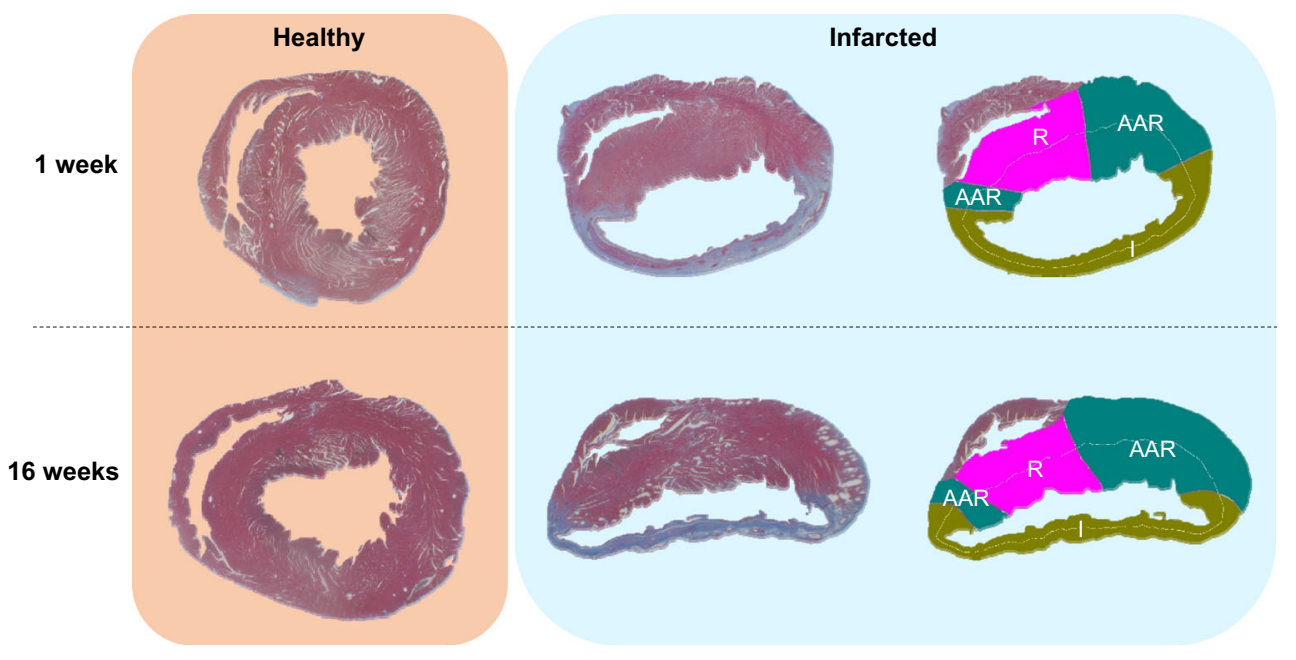


Fig. 4 Inflammatory heat map representing the hierarchical clustering of TRIUMPH patients and healthy subjects measured by membrane-based technique. The normalized intensity of the inflammatory mediators has been color coded according to the legend (red to green, low to high). Each row indicates the inflammatory profile of an individual. The category (heart failure or healthy) to which each individual is assigned is color coded as blue for heart failure or peach for healthy

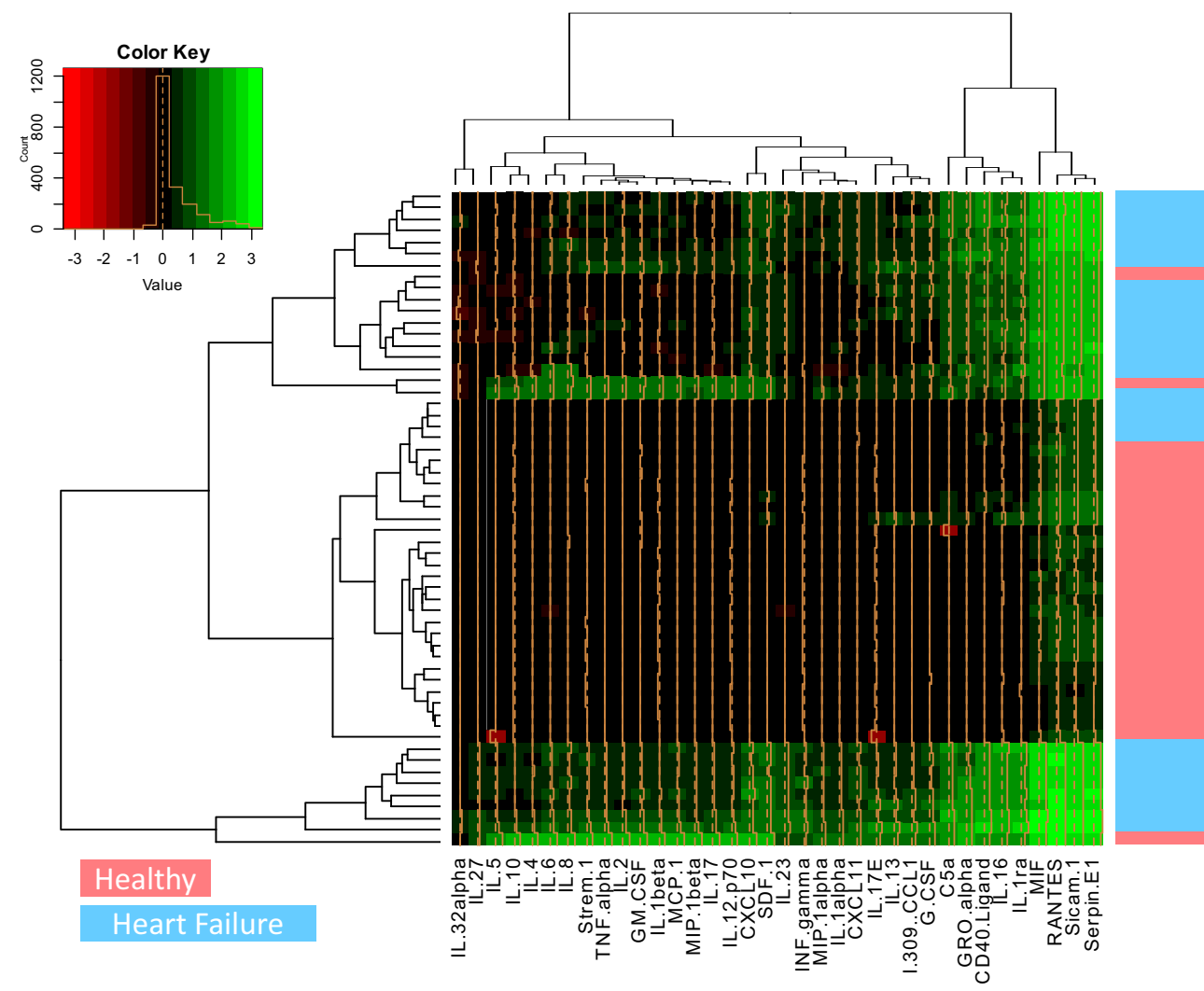

patients in either CXCL10 or NT-proBNP levels (Supplementary Figure 3A and B).

\section{The Relation of CXCL10 with the Heart Failure Severity}

The conventional way to monitor progression of heart failure in patients is the NYHA classification system. We compared CXCL10 concentrations in patients based on their NYHA class. Patients from the TRIUMPH cohort belonged either to class II or classes III-IV. We included data from the FLEMENGHO cohort for additional controls and patients with subclinical asymptomatic heart failure and left ventricular dysfunction (class I). As shown in Fig. 9, CXCL10 concentrations increased in patients with advanced stages of heart failure in both the TRIUMPH and FLEMENGHO cohorts. Of

Fig. 5 Principal component analysis (PCA) of the circulating inflammatory profile of healthy subjects and heart failure patients from the TRIUMPH cohort. The expression levels obtained by the membranebased technique output are analyzed here. a Individuals factor map. The PCA determined the distribution of each individual in a threedimensional space. Each individual was color coded as peach or blue representing a healthy subject or heart failure patient, respectively. In the graph, it is shown how the two groups separate almost completely into two clusters, the healthy subjects and the heart failure patients. b Variables factor map. PCA analysis revealed that MIP- $1 \alpha$ and CXCL10 (red stars) were the best inflammatory mediators for the component 1 in order to discriminate between the two groups. For component 2, CD40 ligand (red dagger) resulted as the best parameter among the highest MFIs to separate the two groups 
a

Individuals factor map - PCA
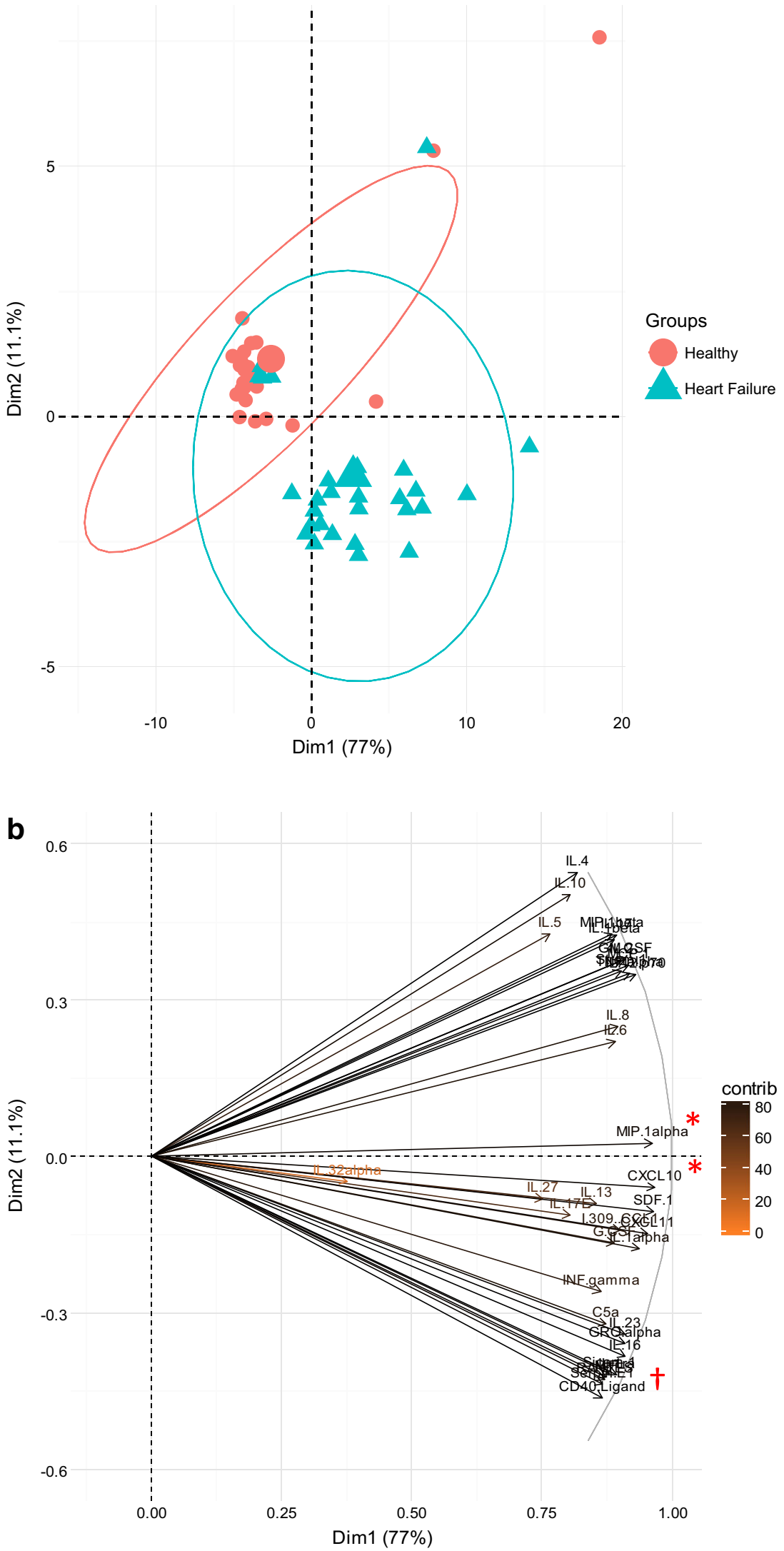
Table 2 Sensitivity and specificity of the top five analytes determined by principal component analysis

\begin{tabular}{llllll}
\hline & Cut-off point (MFI) & Sensitivity \% & $95 \%$ CI & Specificity \% & 95 \% CI \\
\hline MIP-1 $\alpha$ & $>0.89$ & 90 & 73.47 to 97.89 & 89.66 & 72.65 to 97.81 \\
CXCL10 & $>1.85$ & 83.33 & 65.28 to 94.36 & 89.66 & 72.65 to 97.81 \\
CD40 ligand & $>2.76$ & 86.67 & 69.28 to 96.24 & 89.66 & 72.65 to 97.81 \\
IL-4 & $>0.83$ & 70 & 50.60 to 85.27 & 82.76 & 64.23 to 94.15 \\
IL-10 & $>0.83$ & 63.33 & 43.86 to 80.07 & 89.66 & 72.65 to 97.81 \\
\hline
\end{tabular}

Cut-off values for mean florescent intensity (MFI) determined by ROC curve analysis of the top five analytes of Fig. 5b. The table shows the validity of the five analytes determined by membrane-based assay in terms of the tradeoff between sensitivity and specificity. The cut-off point distinguishes healthy from diseased subjects with maximum accuracy note, the relationship between NYHA class and CXCL10 levels held for both cohorts even though CXCL10 was measured by the ELISA technique at separate facilities. When the data were expressed as the relative increase over the mean control value of each study and the two studies combined, circulating CXCL10 levels were significantly increased in patients with symptomatic heart failure, classes II and III (Fig. 9 insert).

No correlation was observed between levels of CXCL10 and either NT-proBNP or C-reactive protein (CRP) (Fig. 10). The latter is a general marker for inflammation and increased plasma levels of CRP are associated with increased risk of cardiovascular diseases, in particular coronary heart disease, as well as poor prognosis in heart failure patients [23, 24]. However, as seen from Fig. 10, a strong positive correlation $(r=0.85 \pm 0.02)$ was observed between CXCL10 and Th1 cytokines (IFN- $\gamma$, IL-2, and TNF $\alpha$ ) and other pro-inflammatory cytokines (MCP1, MIF, MIP-1 $\alpha$, SDF1, CXCL11, IL- $1 \alpha$, and IL-1 $)$. A lower $(p \leq 0.01)$ correlation $(r=0.71 \pm 0.04)$ was

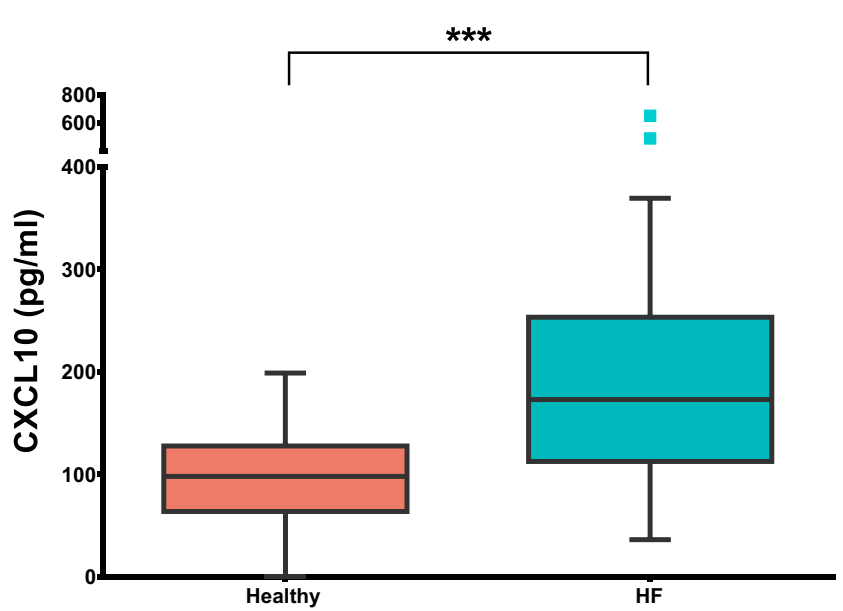

Fig. 6 ELISA measurement of the CXCL10 concentration in healthy subjects and TRIUMPH patients. CXCL10 was measured in serum of healthy individuals and heart failure $(H F)$ patients. The means \pm SEM for healthy and heart failure group are $107.6 \pm 13.7$ and $204.5 \pm 24.37 \mathrm{pg} / \mathrm{mL}$, respectively. Both groups did not pass the normality test (alpha $=0.05$ ). The non-parametric test (Mann-Whitney) revealed that the difference has a significant $p$ value of 0.001 with a confidence level of $99 \%$ observed between CXCL10 and Th2 cytokines (IL-4,-5,-6,$10,-13)$.

\section{Discussion}

Ischemic heart disease and cardiac hypertrophy remain the most important causes of heart failure. There is a large body of evidence that systemic inflammatory pathways are activated in a variety of pathological conditions (e.g., acute MI, hypertension, and atherosclerosis) and that these pathways play a role in the disease progression leading to adverse outcomes [25]. Our understanding of inflammation in heart failure is far from complete; however, several studies indicate that inflammatory mediators might point out the stage of the disease progression. As the need for new biomarkers for a better prognosis, diagnoses, and therapy of heart failure is still strong, we approached this issue making an inventory of cytokines and chemokines involved. Using a rat MI heart failure model, we

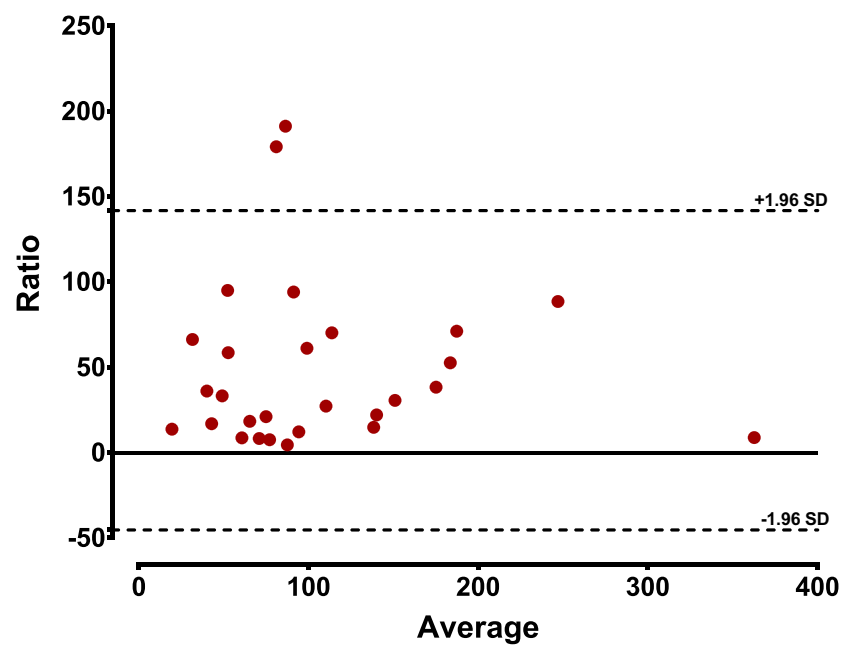

Fig. 7 Comparison of membrane-based immunoassay vs. ELISA for CXCL10 inflammatory profile determination. Bland-Altman analysis was used to determine the correlation quality between the CXCL10 concentration measured by ELISA and the value determined by the membrane-based assay in the TRIUMPH patients. Each of the 28 points on the graph represents an individual patient. The results indicate good correlation within the $95 \%$ confidence 
Fig. 8 Sex difference in CXCL10 concentration in healthy subjects and heart failure $(H F)$ patients enrolled in the TRIUMPH study. Serum values of CXCL10 were analyzed according to male $(M)$ or female $(F)$ category. The non-parametric $t$ test (Mann-Whitney) revealed that CXCL10 concentration was robustly increased in " $\mathrm{F}$ vs. FHF" $(p=0.0033)$ and still significant also in "M-HF vs. M" $(p=0.0290)$.In this graph, the two interquartiles and the median of each category are represented

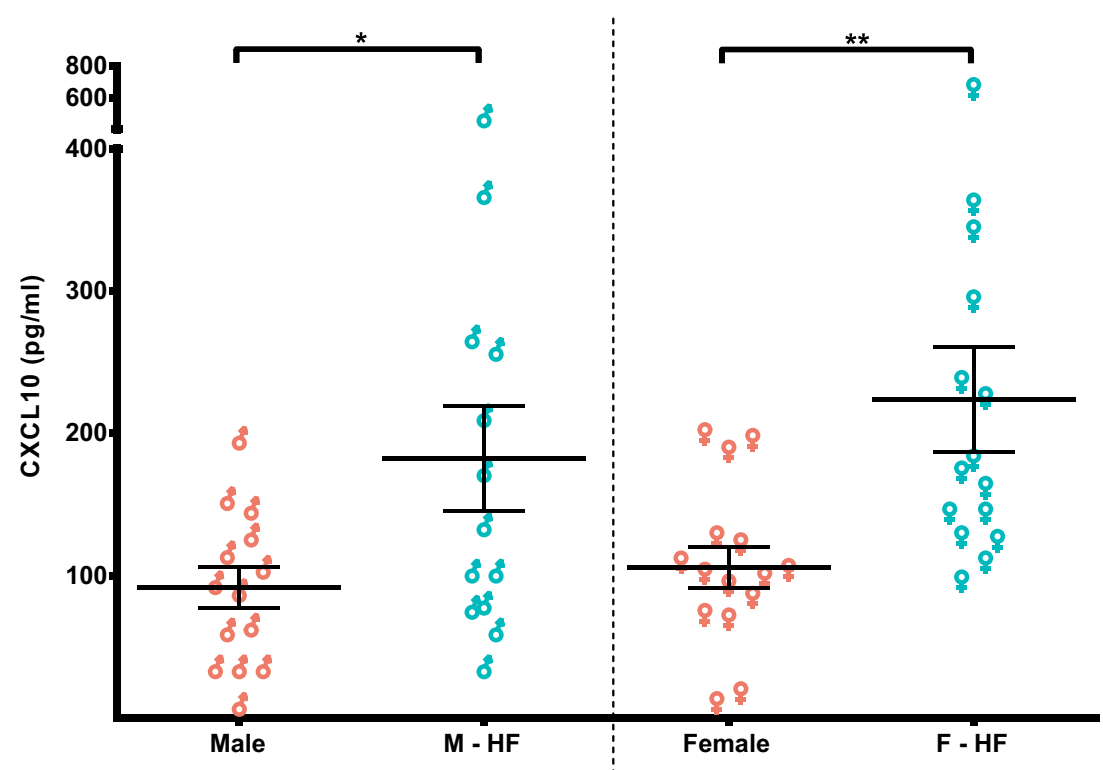

screened for circulating inflammatory mediators in the passage from a healthy to disease status.

In MI rats, permanent occlusion of the LAD resulted in large transmural infarcts with thinning of the left ventricular myocardium without involving the septum and the right ventricle. Infarcts had sizes greater than $35 \%$ of the total LV area. Others observed that cardiac remodeling takes place immediately after coronary artery ligation and acute inflammation dissipates in the first week [26]. Thus, we selected 1 week after MI surgery. Besides, we wanted to be sure that inflammatory mediators, whenever determined, were solely triggered by the inflammatory response due to the cardiac insult [27], disregarding the acute phase attributed to healing from the surgical operation. We observed that circulating levels of CXCL10 and CXCL9 were elevated at 1 week, as were cardiac levels in the remote and infarcted regions. Cardiac levels of CXCL10 remained elevated at 16 weeks in both the infarcted and remote regions. In contrast to CXCL9, CXCL10 was

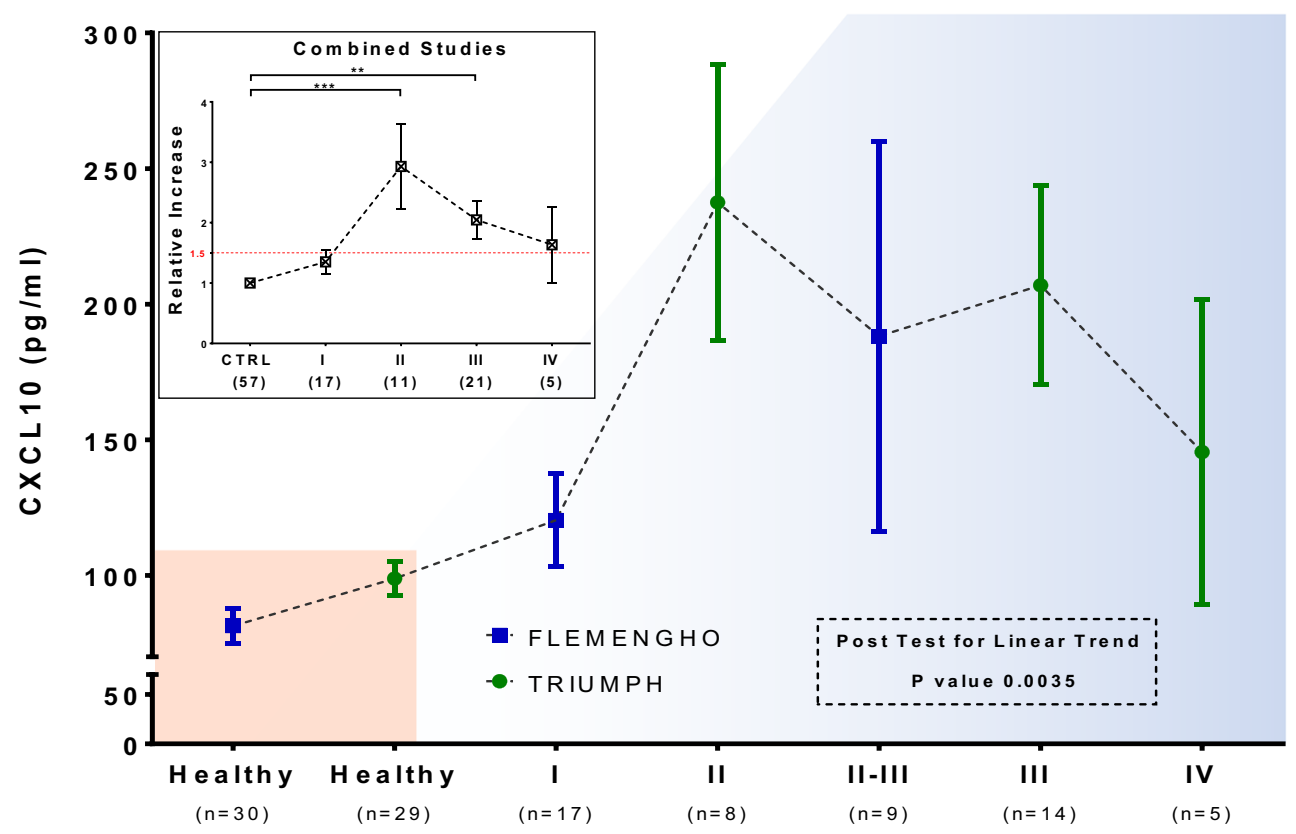

Fig. 9 Distribution of circulating CXCL10 concentration in the TRIUMPH and FLEMENGHO studies according to NYHA classes. Serum CXCL10 measurements for the TRIUMPH cohort are included with those previously published values for the FLEMENGHO cohort so as to include NYHA classes of early stage heart failure progression (asymptomatic and symptomatic, NYHA class I and classes II-III,

respectively). CXCL10 concentrations increased with increasing NYHA class. Insert: The relative increase over control (CTRL) in circulating CXCL10 levels for individual patients grouped by NYHA heart failure class was calculated in order to combine measurements of both studies. $* * p=0.01, * * * p=0.0001$ 


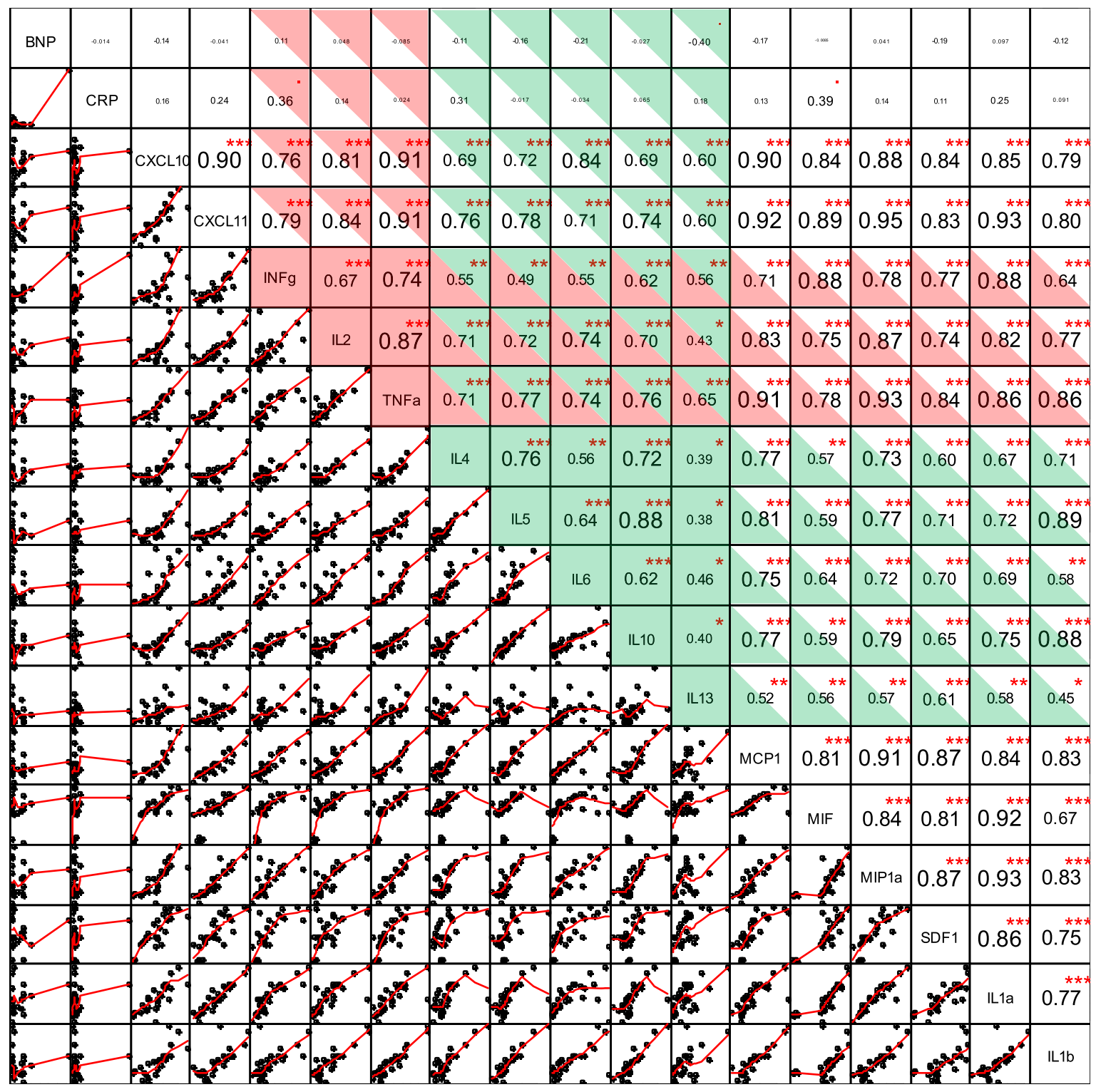

Fig. 10 Multiple linear regression analysis of Th1, Th2, and proinflammatory cytokines in heart failure patients. Linear regression analysis was performed by R statistical software. Numbers in the row to the right of each analyte (center diagonal boxes) provide the correlation coefficient $(r)$ for the extent of linearity with the analyte identified at the

more increased in the infarcted region than the remote region 1 week post-MI. There is a limitation to extending the findings of the ischemic rat model to largely non-ischemic human cohorts; however, our primary purpose for the preclinical study was to demonstrate the upregulation of a pro-inflammatory, so-called driver chemokine in the injured heart, an upregulation that subsisted in heart failure. Additionally, these new results further support our previous study indicating that CXCL10 is also an early marker in pressure-overloaded mouse hearts [14], suggesting that circulating CXCL10 is connected to cardiac remodeling rather than myocardial infarction per se. bottom of that column $\left({ }^{*} p \leq 0.05,{ }^{*} p \leq 0.01\right.$, and ${ }^{* * *} p \leq 0.001$; level of significance is also denoted by font size). Graphic representative of the linear regression analysis appears in the column below each analyte for a particular analyte identified on the right far end of each row

Our study further demonstrates that two cohorts, one of heart failure patients and one of healthy people, can be separated according to their circulating inflammatory profile. Together with our previous findings, we conclude that there is a continuum in the presence of some inflammatory mediators detected in the early stage of heart failure and ultimately in adverse clinical cases in both pre-clinic models and clinic studies $[14,15]$. Heart failure is a syndrome with a broad spectrum of heterogeneous symptoms and clinical features that makes it difficult for the physician to clearly define the state of the patients [28]. It is common practice to classify heart failure patients depending on their symptoms, according 
to the NYHA classes [29]. However, the boundary line to make a distinction between NYHA classes is sometimes very subtle. In addition, genetic and phenotypic variability among patients may force the cardiologist to "customize" the therapy for those patients where the classification fails or is inappropriate. The purpose of the current study was to determine the circulating inflammatory profile together with the expedience of the circulating CXCR3 biomarkers in those patients that belong to NYHA class II and onwards, so-called end-stage heart failure. Although the population size was small, this study demonstrates that healthy and heart failure patients can be segregated solely on hierarchical cluster analysis of the subject's inflammatory profile determined by the membrane-based method [20, 30]. The heatmap in Fig. 4 provided us with a graphical representation of a high dimension data set. A way to identify "key markers" responsible for segregation was appropriate. Hence, PCA was used as a powerful statistical tool for such analysis. PCA disentangled the data, revealing that MIP- $1 \alpha$, CXCL10 (for component 1), and CD40 ligand (for component 2) were inflammatory mediators that guided discrimination between the heart failure patients and healthy individuals.

MIP- $1 \alpha$, also known as CCL3, is normally involved in recruitment and activation of polymorphonuclear leukocytes in the acute inflammatory state. Its general role is not yet elucidated and a link to cardiovascular diseases is far from clear. Nevertheless, Blossom et al. [31] identified a maternal cytokine signature in CHD-affected pregnancies. They observed that the immunological response is principally driven by a robust increase of circulating MIP- $1 \alpha$, suggesting skewing toward an M1 macrophage response in CHDaffected mothers. The functional role of resident macrophages in the heart is not fully understood. However, consistent evidence reports that M1 macrophages are involved in the cardioprotective mechanisms of the heart targeting the neovascularization and inducing debris clearance [32]. The role of CD40 ligand in cardiovascular disease is also far from clear. However, there is an increasing body of evidence that connects the presence of CD40 ligand to heart-related problems such as vascular dysfunction/inflammation [33], coronary heart disease [34], and cardiac allograft rejection [35, 36].

We did not observe any correlation between serum levels of CXCL10 and NT-proBNP or CRP (Fig. 10). Increased levels of NT-proBNP or CRP are associated with heart failure [24]. However, both NT-proBNP and CRP are general end-markers of cardiac stress (wall stress and inflammation, respectively). In contrast to the lack of causality associated with these biomarkers, CXCL10 as an attractant for pro-inflammatory Th1 lymphocytes is a driver of inflammation [15] and might be construed as a more refined measure of cardiac stress. Consistent with this idea is our observation of a strong correlation between levels of CXCL10 and Th1 and proinflammatory cytokines (Fig. 10). The pro-inflammatory role of CXCL10 might provide prognostic utility to this chemokine that is not provided by either BNP or CRP [13, 23, 24, 37].

We recently reported increased circulating CXCL10 levels in patients with diastolic LV dysfunction due to hypertension $[14,15]$. To further extend this observation, we determined its concentration in end-stage heart failure patients. As shown in Figs. 6 and 9, CXCL10 levels were significantly increased in heart failure patients when compared to healthy people. Circulating CXCL10 increased with increasing NYHA classification I to IV, with significant changes in patients with symptomatic heart failure (Fig. 9). With regard to sex differences, a sub-analysis of the subjects (Fig. 8) revealed that females with heart failure have substantially elevated levels of circulating CXCL10. However, the design of the study might have influenced this outcome: (1) females included in class III are more than males, (2) the etiology of the two subgroups is not homogeneous, and (3) sample size is limited. The latter point is of course a limitation of this pilot study. On the other hand, this study permitted investigation of a panel of promising biomarkers [15], creating the basis for further validation in a larger cohort. These biomarkers may ultimately be interpolated in a new classification system that is capable of categorizing patients (minimally invasively) and giving the physician a more reliable instrument to determine level and degree of heart failure. Finally, such a tool could also help the physician manage those patients that need personalized therapy in the most critical stage of heart failure.

\section{Conclusions}

A considerable amount of literature has recently been published on inflammation in heart failure. This study confirms once more that inflammatory mediators are circulating in heart failure patients and the emerging importance of CXCR3 ligands in cardiovascular diseases [15]. The presence of CXCL10, MIP$1 \alpha$, and CD40 ligand appears to be dominant in a population of 38 heart failure patients with advanced heart failure. The circulating levels of CXCL10, which we already demonstrated to be elevated in a subclinical population of patients with LV diastolic dysfunction, were increased in heart failure patients with higher NYHA classification. Due to the small patient number used in the present study, more research on larger cohorts needs to be undertaken before the association between CXCL10 and heart failure clinical parameters is understood more clearly.

Acknowledgments The authors would like to thank Dr. F.A. Zouein for the thoughtful discussion on this study. We are appreciative of the outstanding contribution of Dr. Joke Orsel in helping with the ELISA assay.

\section{Compliance with Ethical Standards}

Conflict of Interest The authors declare that they have no conflict of interest. 
Human Subjects/Informed Consent The recruitment of subjects was performed according to the Medical Ethical Committees of Groningen and Maastricht University, The Netherlands, and in accordance with of the Declaration of Helsinki of 1975.

Animal Studies All experimental and surgical procedures were approved by the Institutional Council on Animal Care and Use of the Maastricht University and complied with the National Institutes of Health Guide for the Care and Use of Laboratory Animals.

Sources of Funding This research was performed within the framework of CTMM, the Center for Translational Molecular Medicine (www.ctmm.nl), project TRIUMPH grant 01C-103, and supported by the Dutch Heart Foundation.

\section{References}

1. Sidney, S., Rosamond, W. D., Howard, V. J., Luepker, R. V., National Forum for Heart, D, \& Stroke, P. (2013). The "heart disease and stroke statistics-2013 update" and the need for a national cardiovascular surveillance system. Circulation, 127(1), 21-23. doi:10.1161/CIRCULATIONAHA.112.155911.

2. Moreno, V., Hernandez-Romero, D., Vilchez, J. A., GarciaHonrubia, A., Cambronero, F., Casas, T., et al. (2010). Serum levels of high-sensitivity troponin T: a novel marker for cardiac remodeling in hypertrophic cardiomyopathy. Journal of Cardiac Failure, 16(12), 950-956. doi:10.1016/j.cardfail.2010.07.245.

3. Gaggin, H. K., \& Januzzi, J. L., Jr. (2013). Biomarkers and diagnostics in heart failure. Biochimica et Biophysica Acta, 1832(12), 2442-2450. doi:10.1016/j.bbadis.2012.12.014.

4. Montoro-Garcia, S., Hernandez-Romero, D., Jover, E., GarciaHonrubia, A., Vilchez, J. A., Casas, T., et al. (2012). Growth differentiation factor-15, a novel biomarker related with disease severity in patients with hypertrophic cardiomyopathy. European Journal of Internal Medicine, 23(2), 169-174. doi:10.1016/j.ejim. 2011.08.022.

5. Maisel, A., Mueller, C., Nowak, R., Peacock, W. F., Landsberg, J. W., Ponikowski, P., et al. (2010). Mid-region pro-hormone markers for diagnosis and prognosis in acute dyspnea: results from the BACH (Biomarkers in Acute Heart Failure) trial. Journal of the American College of Cardiology, 55(19), 2062-2076. doi:10. 1016/j.jacc.2010.02.025.

6. Pfisterer, M., Buser, P., Rickli, H., Gutmann, M., Erne, P., Rickenbacher, P., et al. (2009). BNP-guided vs symptom-guided heart failure therapy: the Trial of Intensified vs Standard Medical Therapy in Elderly Patients with Congestive Heart Failure (TIMECHF) randomized trial. JAMA, 301(4), 383-392. doi:10.1001/ jama.2009.2.

7. Dickstein, K., Cohen-Solal, A., Filippatos, G., McMurray, J. J., Ponikowski, P., \& Poole-Wilson, P. A. (2008). ESC guidelines for the diagnosis and treatment of acute and chronic heart failure 2008: the Task Force for the diagnosis and treatment of acute and chronic heart failure 2008 of the European Society of Cardiology. Developed in collaboration with the Heart Failure Association of the ESC (HFA) and endorsed by the European Society of Intensive Care Medicine (ESICM). European Journal of Heart Failure, 10(10), 933-989. doi:10.1016/j.ejheart.2008.08.005.

8. Desai, A. S. (2013). Are serial BNP measurements useful in heart failure management? Serial natriuretic peptide measurements are not useful in heart failure management: the art of medicine remains long. Circulation, 127(4), 509-516. doi:10.1161/ CIRCULATIONAHA.112.120493. discussion 516.

9. Rodeheffer, R. J. (2004). Measuring plasma B-type natriuretic peptide in heart failure: good to go in 2004? Journal of the American College of Cardiology, 44(4), 740-749. doi:10.1016/j.jacc.2004. 03.082 .

10. Richards, A. M. (2007). Variability of NT-proBNP levels in heart failure: implications for clinical application. Heart, 93(8), 899-900. doi:10.1136/hrt.2006.110643.

11. Boerrigter, G., Costello-Boerrigter, L. C., \& Burnett, J. C., Jr. (2009). Natriuretic peptides in the diagnosis and management of chronic heart failure. Heart Failure Clinics, 5(4), 501-514. doi: 10.1016/j.hfc.2009.04.002.

12. Madamanchi, C., Alhosaini, H., Sumida, A., \& Runge, M. S. (2014). Obesity and natriuretic peptides, BNP and NT-proBNP: mechanisms and diagnostic implications for heart failure. International Journal of Cardiology, 176(3), 611-617. doi:10. 1016/j.ijcard.2014.08.007.

13. Writing Committee, M., Yancy, C. W., Jessup, M., Bozkurt, B., Butler, J., Casey, D. E., Jr., et al. (2013). 2013 ACCF/AHA guideline for the management of heart failure: a report of the American College of Cardiology Foundation/American Heart Association Task Force on practice guidelines. Circulation, 128(16), e240 327. doi:10.1161/CIR.0b013e31829e8776.

14. Altara, R., Gu, Y. M., Struijker-Boudier, H. A., Thijs, L., Staessen, J. A., \& Blankesteijn, W. M. (2015). Left ventricular dysfunction and CXCR3 ligands in hypertension: from animal experiments to a population-based pilot study. PloS One, 10(10), e0141394. doi:10. 1371/journal.pone.0141394.

15. Altara, R., Manca, M., Brandao, R.D., Zeidan, A., Booz, G.W., \& zouein, F. A. (2016). Emerging importance of chemokine receptor CXCR3 and its ligands in cardiovascular diseases. Clinical Science (London, England: 1979), 130(7).

16. Daskalopoulos, E.P., Vilaeti, A.D., Barka, E., Mantzouratou, P., Kouroupis, D., Kontonika, M., et al. (2015). Attenuation of postinfarction remodeling in rats by sustained myocardial growth hormone administration. Growth Factors:1-9.

17. Higuchi, T., Nekolla, S. G., Jankaukas, A., Weber, A. W., Huisman, M. C., Reder, S., et al. (2007). Characterization of normal and infarcted rat myocardium using a combination of small-animal PET and clinical MRI. Journal of Nuclear Medicine, 48(2), 288294.

18. Kuznetsova, T., Mischak, H., Mullen, W., \& Staessen, J. A. (2012). Urinary proteome analysis in hypertensive patients with left ventricular diastolic dysfunction. European Heart Journal, 33(18), 2342-2350. doi:10.1093/eurheartj/ehs185.

19. Kuznetsova, T., Herbots, L., Lopez, B., Jin, Y., Richart, T., Thijs, L., et al. (2009). Prevalence of left ventricular diastolic dysfunction in a general population. Circulation. Heart Failure, 2(2), 105-112. doi: 10.1161/CIRCHEARTFAILURE.108.822627.

20. Altara, R., Manca, M., Hessel, M. H., Janssen, B. J., StruijkerBoudier, H. H., Hermans, R. J., et al. (2014). Improving membrane based multiplex immunoassays for semi-quantitative detection of multiple cytokines in a single sample. BMC Biotechnology, 14, 63 . doi:10.1186/1472-6750-14-63.

21. Battes, L. C., Caliskan, K., Rizopoulos, D., Constantinescu, A. A., Robertus, J. L., Akkerhuis, M., et al. (2015). Repeated measurements of NT-pro-B-type natriuretic peptide, troponin T or Creactive protein do not predict future allograft rejection in heart transplant recipients. Transplantation, 99(3), 580-585. doi:10. 1097/TP.0000000000000378.

22. Bernhard, O. K., Mathias, R. A., Barnes, T. W., \& Simpson, R. J. (2011). A fluorescent microsphere-based method for assay of multiple analytes in plasma. Methods in Molecular Biology, 728, 195 206. doi:10.1007/978-1-61779-068-3 12. 
23. Huynh, K., Van Tassell, B., \& Chow, S. L. (2015). Predicting therapeutic response in patients with heart failure: the story of Creactive protein. Expert Review of Cardiovascular Therapy, 13(2), 153-161. doi:10.1586/14779072.2015.1000307.

24. Savarese, G., Rosano, G. M., Parente, A., D'Amore, C., Reiner, M. F., Camici, G. G., et al. (2014). Reduction of C-reactive protein is not associated with reduced cardiovascular risk and mortality in patients treated with statins. A meta-analysis of 22 randomized trials. International Journal of Cardiology, 177(1), 152-160. doi: 10.1016/j.ijcard.2014.09.028.

25. Altara, R., Manca, M., Sabra, R., Eid, A. A., Booz, G. W., \& Zouein, F. A. (2015). Temporal cardiac remodeling postmyocardial infarction: dynamics and prognostic implications in personalized medicine. Heart Failure Reviews. doi:10.1007/ s10741-015-9513-8.

26. Schoemaker, R. G., Debets, J. J., Struyker-Boudier, H. A., \& Smits, J. F. (1991). Delayed but not immediate captopril therapy improves cardiac function in conscious rats, following myocardial infarction. Journal of Molecular and Cellular Cardiology, 23(2), 187-197.

27. Yan, X., Shichita, T., Katsumata, Y., Matsuhashi, T., Ito, H., Ito, K., et al. (2012). Deleterious effect of the IL-23/IL-17A axis and gammadeltaT cells on left ventricular remodeling after myocardial infarction. Journal of the American Heart Association, 1(5), e004408. doi:10.1161/JAHA.112.004408.

28. Raphael, C., Briscoe, C., Davies, J., Ian Whinnett, Z., Manisty, C., Sutton, R., et al. (2007). Limitations of the New York Heart Association functional classification system and self-reported walking distances in chronic heart failure. Heart, 93(4), 476-482. doi: 10.1136/hrt.2006.089656.

29. Goldman, L., Hashimoto, B., Cook, E. F., \& Loscalzo, A. (1981). Comparative reproducibility and validity of systems for assessing cardiovascular functional class: advantages of a new specific activity scale. Circulation, 64(6), 1227-1234.

30. Altara, R., Manca, M., Hermans, K. C., Daskalopoulos, E. P., Brunner-La Rocca, H. P., Hermans, R. J., et al. (2015). Diurnal rhythms of serum and plasma cytokine profiles in healthy elderly individuals assessed using membrane based multiplexed immunoassay. Journal of Translational Medicine, 13, 129. doi:10.1186/ s12967-015-0477-1.

31. Blossom, S. J., Rau, J. L., Best, T. H., Bornemeier, R. A., \& Hobbs, C. A. (2013). Increased maternal cytokine production and congenital heart defects. Journal of Reproductive Immunology, 97(2), 204210. doi:10.1016/j.jri.2012.12.004.

32. Fujiu, K., Wang, J., \& Nagai, R. (2014). Cardioprotective function of cardiac macrophages. Cardiovascular Research, 102(2), 232 239. doi:10.1093/cvr/cvu059.

33. Hausding, M., Jurk, K., Daub, S., Kroller-Schon, S., Stein, J., Schwenk, M., et al. (2013). CD40L contributes to angiotensin IIinduced pro-thrombotic state, vascular inflammation, oxidative stress and endothelial dysfunction. Basic Research in Cardiology, 108(6), 386. doi:10.1007/s00395-013-0386-5.

34. Kaptoge, S., Seshasai, S. R., Gao, P., Freitag, D. F., Butterworth, A. S., Borglykke, A., et al. (2014). Inflammatory cytokines and risk of coronary heart disease: new prospective study and updated metaanalysis. European Heart Journal, 35(9), 578-589. doi:10.1093/ eurheartj/eht367.

35. Fu, W., Zhu, J., Qiu, Y., \& Li, W. (2013). Induction of CD4+ $\mathrm{CD} 25+\mathrm{T}$ cells and control of cardiac allograft rejection by CD40/ CD40L costimulatory pathway blockade in mice. Transplantation Proceedings, 45(2), 611-617. doi:10.1016/j.transproceed.2012.10. 044.

36. Krill, K. T., Csencsits-Smith, K., Wood, S. C., Faust, S., Lu, G., \& Bishop, D. K. (2013). Glucocorticoid-induced TNFR-related protein stimulation reverses cardiac allograft acceptance induced by CD40-CD40L blockade. Clinical \& Developmental Immunology, 2013, 986859. doi:10.1155/2013/986859.

37. Krintus, M., Kozinski, M., Kubica, J., \& Sypniewska, G. (2014). Critical appraisal of inflammatory markers in cardiovascular risk stratification. Critical Reviews in Clinical Laboratory Sciences, 51(5), 263-279. doi:10.3109/10408363.2014.913549. 